\title{
Calculations of electromagnetic (and a few weak) reactions on light nuclei in chiral effective theory
}

\author{
Daniel R. Phillips* \\ Institute for Nuclear and Particle Physics and \\ Department of Physics and Astronomy, \\ Ohio University, \\ Athens, Ohio 45701, USA \\ Helmholtz-Institut für Strahlen- und Kernphysik (Theorie), \\ Universität Bonn, \\ D-53115, Bonn, Germany \\ E-mail: phillips@phy.ohiou.edu
}

I discuss the calculation of electromagnetic and weak reactions in systems with $A=2-4$ using potentials and current operators derived within chiral effective theory $(\chi \mathrm{ET})$. I focus on computations up to $O\left(P^{3}\right)$ relative to leading, and review results that show:

(a) At this order $\chi \mathrm{ET}$ gives a good description of extant data on the deuteron's charge and quadrupole form factors for momentum transfers $|\mathbf{q}|<0.6 \mathrm{GeV}$. Its predictions will be challenged by forthcoming precision data from BLAST and JLab.

(b) The $\chi \mathrm{ET}$ nuclear force and current operator can be fixed up to $O\left(P^{3}\right)$ using NN-system observables, together with two pieces of strong-interaction data from the three-nucleon system. $\chi \mathrm{ET}$ then explains the magnetic moments of ${ }^{3} \mathrm{H}$ and ${ }^{3} \mathrm{He}$, as well as experiments on the threshold capture reaction $\mathrm{nd} \rightarrow \mathrm{t} \gamma$.

(c) The $\chi$ ET expansion for weak currents has reached unprecedented accuracy. When used to anlayze data on the weak-capture process, ${ }^{3} \mathrm{He}\left(\mu^{-}, v_{\mu}\right)^{3} \mathrm{H}$, it yields the tightest contraint on the conserved vector-current hypothesis.

In all these cases I stress $\chi$ ET's ability to systematically describe the interplay between the pionrange and shorter-distance dynamics that is at work in these reactions.

6th International Workshop on Chiral Dynamics, CD09

July 6-10, 2009

Bern, Switzerland

\footnotetext{
${ }^{*}$ Speaker.
} 


\section{Introduction}

The use of $\chi \mathrm{PT}$ to compute nuclear forces is a problem that has received much attention over the past two decades. Electromagnetic reactions provide the opportunity to probe the accuracy of the wave functions obtained in such computations. In this contribution I will discuss the use of $\chi$ PT potentials and current operators in calculations of electron-scattering, radiative-capture, photodisintegration, and weak reactions with light-nuclear targets.

The framework for these calculations is "chiral effective theory" $(\chi$ ET). In this approach $[1,2]$ $\chi \mathrm{PT}$ is used to compute the NN potential up to some fixed order, $n$, in the chiral expansion in powers of $\left(p, m_{\pi}\right) / \Lambda_{0}$, with the breakdown scale $\Lambda_{0}$ nominally being $m_{\rho} \sim 4 \pi f_{\pi}$, but in actuality being somewhat lower for reactions involving baryons. ( $\Lambda_{0}$ can be extended back to something like the original scale if additional degrees of freedom—in particular the Delta(1232) — are included explicitly in the theory, but I will not discuss such variants of $\chi \mathrm{PT}$ here.) The computation of the current operator for the reaction in question is then also organized as an expansion in powers of $P$, and the pertinent matrix elements constructed via:

$$
\mathscr{M}_{\mu}=\left\langle\psi^{(f)}\left|\sum_{k=0}^{n} J_{\mu}^{(k)}\right| \psi^{(i)}\right\rangle
$$

where $\left|\psi^{(i)}\right\rangle$ and $\left|\psi^{(f)}\right\rangle$ are solutions of the Schrödinger equation for the $\chi$ PT potential V. This allows both an examination of the convergence of the expansion as a function of the maximum order, $n$, of the calculation, and the specification of theoretical uncertainties based on the anticipated size of the $O\left(P^{n+1}\right)$ corrections to the process of interest.

To solve the Schrödinger equation with potentials derived from $\chi \mathrm{PT}$ one must introduce a cutoff, $\Lambda$, on the intermediate states, because the $\chi$ PT potentials grow with momenta. The contact interactions in $\mathscr{L}_{N N}$ should then absorb the dependence of the effective theory's predictions on $\Lambda$ in all low-energy observables. Otherwise we conclude that $\chi \mathrm{ET}$ is unable to give reliable predictions. In Refs. $[2,3,4,5,6] V$ was computed to a fixed order, and then the NN LECs that appear in $V$ were fitted to NN data for a range of cutoffs between 500 and $800 \mathrm{MeV}$. The resulting predictionsespecially the ones obtained with the $O\left(P^{4}\right)$ potential of Refs. [5, 6]—contain very little residual cutoff dependence in this range of $\Lambda$ 's, and describe $\mathrm{NN}$ data with considerable accuracy.

However, several recent papers showed that $\chi \mathrm{ET}$ - at least as presently formulated — does not yield stable leading-order predictions once cutoffs larger than $m_{\rho}(\sim 800 \mathrm{MeV})$ are considered [7, $8,9,10,11,12]$. This suggests that the theory described above is not properly renormalized, i.e. the impact of short-distance physics on the results is not under control. Issues associated with this difficulty are discussed elsewhere in this volume $[13,14,15]$. These contributions make it clear that the approach described in this review-one based on straightforward application of $\chi \mathrm{PT}$ to the NN potential $V$ - cannot be used over a wide range of cutoffs.

It can perhaps be used if we will follow Refs. $[16,17]$ and employ cutoffs $\Lambda$ in the vicinity of $m_{\rho}$. Since the short-distance physics of the effective theory for $p \gg m_{\rho}$ is (presumably) completely different to the short-distance physics of QCD itself, it is not clear that considering larger cutoffs yields any additional information regarding the true impact of short-distance physics on observables. Using low cutoffs in the effective theory also has the advantage that relevant momenta are 
demonstrably within the domain of validity of $\chi$ PT. This justifies $\chi$ ET as a systematic theory, but at the cost of limiting the cutoffs to $m_{\pi} \ll \Lambda<m_{\rho}$.

Calculations of NN scattering using $\Lambda$ 's in this window have been performed in Refs. [2, 3, 4, $5,6]$ with, as noted above, considerable phenomenological success. Consistent three-nucleon and four-nucleon forces have also been derived and implemented in such an approach $[18,19,20]$. Here I will look at $\chi$ ET calculations for electron-deuteron scattering [21, 22, 23, 24], magnetic-moments and capture in the tri-nucleons [25, 27], $\mathrm{n}^{3} \mathrm{He}$ capture [28], Helium-4 photoabsorption [29], and selected weak reactions. I will not discuss Compton scattering from the NN and NNN systems. There is considerable experimental interest in these processes, but the advances in theory are summarized in the contributions to these proceedings by McGovern and Grießhammer [30, 31]. The exciting discovery that elastic Compton scattering from Helium-3 nuclei could be a useful probe of dipole electric and magnetic neutron polarizabiltiies and neutron spin polarizabilities [32, 33] was already discussed at the last "Chiral Dynamics" workshop.

\section{Electron-deuteron scattering}

In Ref. [9] the Schrödginer equation was solved in momentum space using the leading-order (LO) $\chi$ PT potential and cutoffs $\Lambda=0.4-4 \mathrm{GeV}$. That potential consists solely of one-pion exchange, plus a contact interaction. At each $\Lambda$ the strength of the contact interaction was adjusted to reproduce the deuteron binding energy. The consequent renormalization of the $\mathrm{NN}$ interaction is sufficient to render LO predictions in this channel stable with respect to $\Lambda$ up to arbitrarily high cutoffs [7, 34]. Ref. [24] then used the resulting deuteron wave functions to calculate electromagnetic deuteron form factors via the standard (Breit-frame) formulae (see e.g. Ref. [23]):

$$
\begin{gathered}
G_{C}=\frac{1}{3|e|}\left(\left\langle 1\left|J^{0}\right| 1\right\rangle+\left\langle 0\left|J^{0}\right| 0\right\rangle+\left\langle-1\left|J^{0}\right|-1\right\rangle\right), \\
G_{Q}=\frac{1}{2|e| \eta M_{d}^{2}}\left(\left\langle 0\left|J^{0}\right| 0\right\rangle-\left\langle 1\left|J^{0}\right| 1\right\rangle\right), \quad G_{M}=-\frac{1}{\sqrt{2 \eta}|e|}\left\langle 1\left|J^{+}\right| 0\right\rangle,
\end{gathered}
$$

where we have labeled the (non-relativistic) deuteron states by the projection of the deuteron spin along the direction of the momentum transfer $\mathbf{q}$ and $\eta \equiv|\mathbf{q}|^{2} /\left(4 M_{d}^{2}\right) . G_{C}, G_{Q}$, and $G_{M}$ are related to the experimentally-measured $A, B$, and $T_{20}$ in the usual way, with $T_{20}$ being primarily sensitive to $G_{Q} / G_{C}$ and $B$ depending only to $G_{M}$.

Here we will compare $\chi$ ET predictions for $G_{C}$ and $G_{Q}$ with extractions from data for the deuteron structure function $A$ and the tensor-polarization observable $T_{20}$ [35]. For this purpose we use the deuteron charge operator

$$
\left\langle\mathbf{p}^{\prime}\left|J_{0}(\mathbf{q})\right| \mathbf{p}\right\rangle=|e| \boldsymbol{\delta}^{(3)}\left(p^{\prime}-p-q / 2\right) G_{E}^{(s)}\left(Q^{2}\right),
$$

with $G_{E}^{(s)}$ the nucleon's isoscalar electric form factor. This is the $O(e)$ result for $J_{0}$, but it holds up to corrections suppressed by $P^{3}$ (apart from some small effects that have coefficients $\sim 1 / M^{2}$ ).

The results are shown in the left panel of Fig. 1. Note that the calculation contains no free parameters beyond the one NN LEC that is adjusted to ensure that the deuteron binding energy is reproduced. Even though we are outside the range of cutoffs where the standard $\chi \mathrm{PT}$ power counting can be expected to apply, as $\Lambda \rightarrow \infty$ the momentum-space wave functions produce a $G_{C}$ 
that converges to a definite result. The agreement with experimental data at low- $|\mathbf{q}|$ is quite good, but the LO wave functions predict a minimum in $G_{C}$ at too large a $|\mathbf{q}|^{2}$. The result is even better for $G_{Q}$ (not shown), where the agreement with data is excellent to a surprisingly large value of $|\mathbf{q}|$.
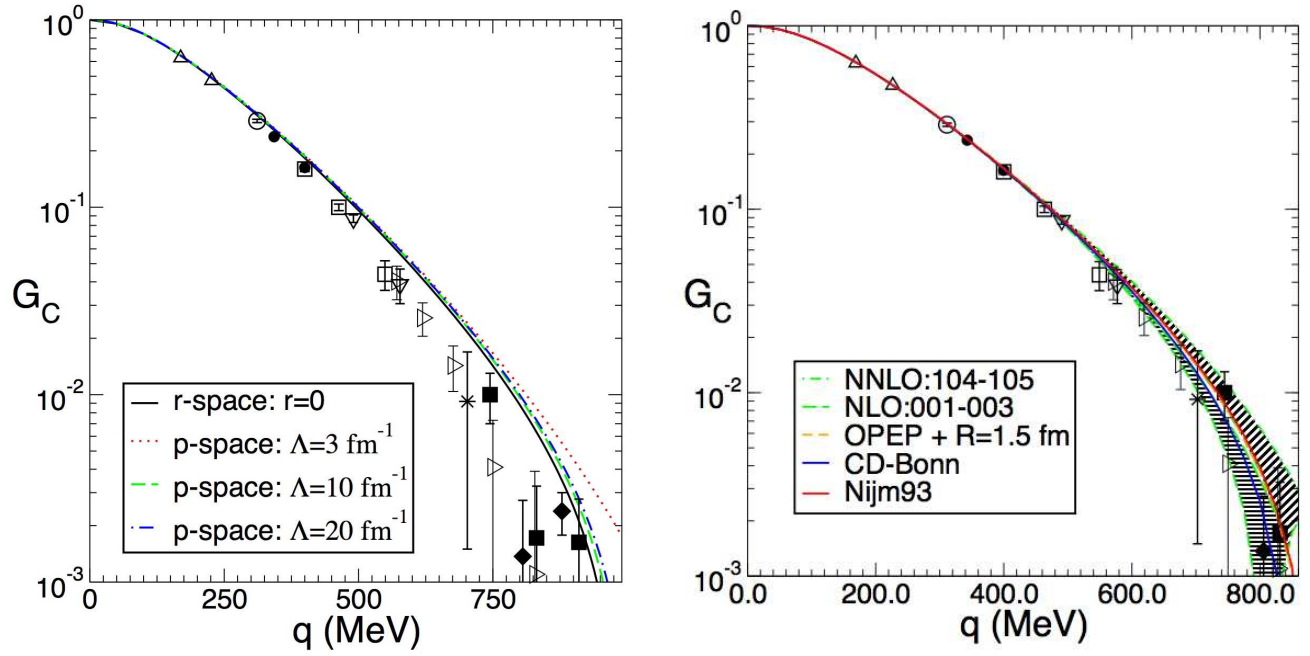

Figure 1: Predictions for $G_{C}$ with $\mathrm{LO}$ wave functions (left panel) and various (low-cutoff) wave functions, most of which include two-pion exchange (right panel). Data from Refs. [35, 36]. Left panel from Ref. [24].

To go beyond LO we must consider corrections to the $N N$ potential $V$, and to the charge operator $J_{0}$. The right panel of Fig. 1 shows the evaluation of $G_{C}$ to three orders beyond leading, $O\left(e P^{3}\right)$, for a variety of different wave functions, including the $\chi \mathrm{ET}$ wave function of consistent (next-to-next-to-leading $\left[O\left(P^{3}\right)\right]$ ) order. In these calculations all cutoffs are kept below $1 \mathrm{GeV}$, so as to ensure that the $O\left(P^{2}\right)$ and $O\left(P^{3}\right)$ corrections to the deuteron wave function are only small perturbations to the $\mathrm{LO}$ result. The range of predictions for $G_{C}$ in $\chi \mathrm{ET}$ at $O\left(e P^{3}\right)$ is indicated by the diagonally shaded band. It is clear that this prediction is in signficantly better agreement with the electron-deuteron scattering data than is the LO one, indicating that the two-pion exchange corrections to the NN potential help to get the minimum of the deuteron $G_{C}$ in the correct place.

Turning our attention to the quadrupole structure of deuterium, we now consider $G_{C} / G_{Q}$. At $O\left(e P^{3}\right)$ we find that the quadrupole moment, $Q_{d}$, is somewhat sensitive to the short-distance physics included in the deuteron wave function, varying by $2 \%$ when the cutoff in the $N N$ system is changed by $\sim 100 \%$. Intriguingly, this is roughly the magnitude of the discrepancy between the $Q_{d}$ predicted at this order and the experimental value $Q_{d}=0.2859(3) \mathrm{fm}^{2}$. There are no isoscalar corrections to $J_{0}$ at $O\left(e P^{4}\right)$ [37, 38]. Thus, Ref. [23] considered the dominant effect at $O\left(e P^{5}\right)$ : a short-distance operator that represents the contribution of modes above $\Lambda_{0}$ to $G_{Q}$. Matrix elements of this operator vary more slowly with $|\mathbf{q}|^{2}$ than do the effects due to the one-body mechanisms that give the LO contribution to $G_{Q}$. Therefore we can constrain the impact of the short-distance $O\left(e P^{5}\right)$ mechansim by demanding that its coefficient be such that the experimental $Q_{d}$ is reproduced. When this is done we can no longer predict $G_{Q}$ at $Q^{2}=0$, but we can predict the $|\mathbf{q}|^{2}$-dependence of $G_{Q}$. The prediction's theoretical uncertainty comes from the $|\mathbf{q}|^{2}$-dependence of short-distance NN physics, and is $\sim 3 \%$ at $|\mathbf{q}|=2 \mathrm{fm}^{-1}$ (see Fig. 2). The new short-distance quadrupole operator leads to $\chi \mathrm{ET}$ predictions for $G_{C} / G_{Q}$ that have a different $|\mathbf{q}|^{2}$-dependence to 
what is obtained in potential models. BLAST data will provide a significant test of this approach to deuteron electromagnetic structure. Analogous calculations can also easily be performed for $A\left(Q^{2}\right)$, so as to confront forthcoming JLab data [38].

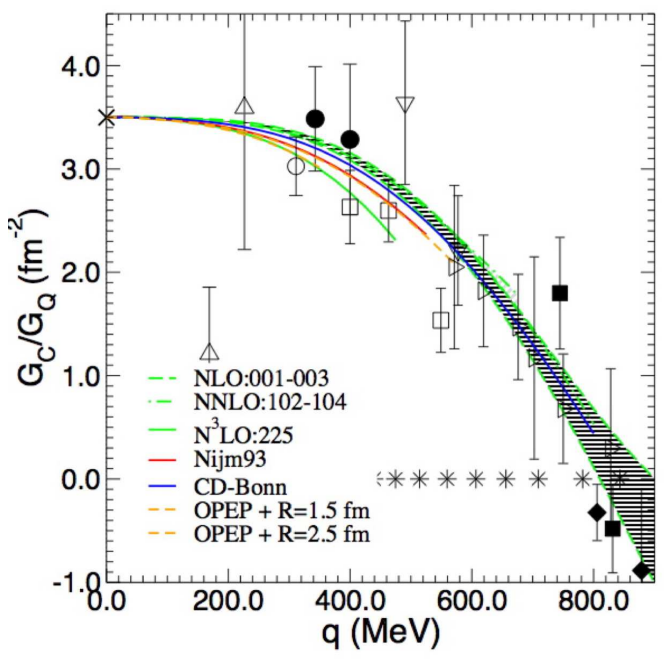

Figure 2: Predictions for $G_{C} / G_{Q}$ from various different deuteron wave functions calculated using $\chi \mathrm{ET}$ and phenomenological potentials. All calculations have a short-distance piece of $J_{0}$ included such that the deuteron quadrupole moment is reproduced. The band represents the theoretical uncertainty and the stars are the values of $|\mathbf{q}|$ where BLAST has data. The data shown is from Refs. [35, 36]. See Ref. [23] for details.

\section{Magnetic structure}

Meanwhile the vector part of the current operator, $\mathbf{J}$ has now been worked out up to $O\left(e P^{4}\right)$. The leading-order $(O(e P))$ part is the standard one-body convection current, together with the single-nucleon magnetic-moment operators. The next-to-leading order corrections to this are purely isovector. They are one-pion-exchange current contributions to $\mathbf{J}$ that are necessary to maintain current conservation. One-loop corrections to the isovector nucleon form factor also enter at the same order. At $O\left(e P^{3}\right)$ sub-leading nucleon structure effects occur.

At $O\left(e P^{4}\right)$ a variety of mechanisms enter (see Fig. 3). Two-body currents, connected to the NLO two-pion exchange of the $\chi$ PT NN potential by minimal substitution, appear. Also present are one-pion-exchange range currents resulting from (a) loop corrections, and (b) insertions from $\mathscr{L}_{\pi N}^{(3)}$. The $O\left(e P^{4}\right)$ current was derived for a calculation of $\vec{n} \mathrm{p} \rightarrow \mathrm{d} \gamma$ in Ref. [39], but the derivation applies only for $\omega \sim|\mathbf{q}| \sim m_{\pi}^{2} / M$. More recently it has been re-derived by two different sets of authors, and extended to the case $\omega \sim m_{\pi}^{2} / M,|\mathbf{q}| \sim m_{\pi}[37,40]$. These papers involve a careful treatment of reducible diagrams, in two different formalisms, and the results for the two-pion-exchange currents agree. (There are, however, some interesting differences with Ref. [39].) Questions remain over the one-pion-exchange range parts of the currents derived in Ref. [40], which need to be checked for consistency as regards renormalization. Crucially, also present at $O\left(e P^{4}\right)$ are two short-distance operators, that encode the coupling of magnetic photons to short-distance components of the NN wave function. The LECs that multiply these operators must be fixed from experimental data. 


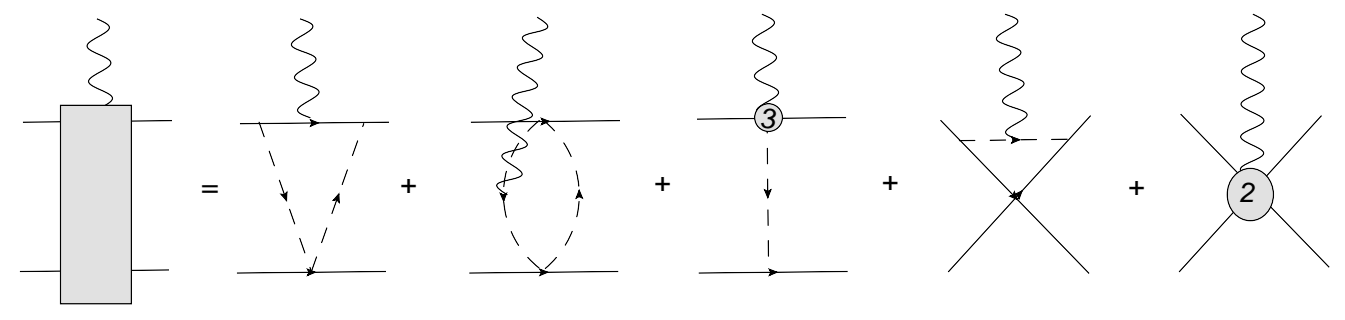

Figure 3: Some contributions to $\mathbf{J}$ at $O\left(e P^{4}\right)$. Dots represent vertices from $\mathscr{L}_{\pi N}^{(1)}$, while vertices with an $n$ inside them are from the pertinent $n$ th-order Lagrangian.

\begin{tabular}{|c|c|c|c|c|}
\hline Cumulative to & $\mu_{d}$ (m.m.) & $M_{n p}\left(\mathrm{fm}^{1 / 2}\right)$ & $\mu_{3_{H}}$ (n.m.) & $\mu_{3_{H e}}$ (n.m.) \\
\hline$O(e P)$ & 0.8469 & 393.1 & 2.585 & -1.774 \\
$O\left(e P^{2}\right)$ & 0.8469 & 401.8 & 2.790 & -1.979 \\
$O\left(e P^{3}\right)$ & 0.8400 & 401.7 & 2.772 & -1.986 \\
$O\left(e P^{4}\right)$ & Fit & Fit & $3.035(12)$ & $-2.198(12)$ \\
\hline Experiment & 0.8574 & $410.2(4)$ & 2.979 & -2.128 \\
\hline
\end{tabular}

Table 1: Deuteron magnetic moment, threshold np capture matrix element, and triton and Helium-3 magnetic moments, at different orders for the operator $\mathbf{J}$. The calculations were done with the AV18 NN potential, supplemented, where appropriate, by the UIX NNN potential. Result from Ref. [25].

The obvious place to do that is in the $\mathrm{A}=2$ system, using the threshold $\mathrm{np} \rightarrow \mathrm{d} \gamma$ capture rate, and the deuteron magnetic moment. The convergence of these two observables as calculated in Ref. [25] is shown in the second and third columns of Table 3. The use of phenomenological potentials means the operator $\mathbf{J}$ is not consistent with the potential $V$ used to compute deuteron and tri-nucleon structure. Some issues, e.g. a lack of exact current conservation, result from this inconsistency. However, such a "hybrid approach" has proven quite successful in other reactions, perhaps because much of the difference between the short-distance pieces of the $\chi \mathrm{ET}$ wave functions and those of the AV18 potential is absorbed in the $O\left(e P^{4}\right)$ short-distance NN magnetic LECs, which are then adjusted so as to reproduce $\mu_{d}$ and $M_{n p}$. Nevertheless, repeating these calculations with consistent wave functions and potentials will be an important future step (see Ref. [40] for efforts in this direction). For the moment, though, this fixes the current up to $O\left(e P^{4}\right)$. The resulting $\mathbf{J}$ can then be applied to capture and magnetic-moment calculations in higher-body systems.

Ref. [25] did this in the $A=3$ system. The key point here is that the three-nucleon current operator does not appear until $O\left(e P^{5}\right)$, and the first order at which an LEC would need to be fixed from $3 \mathrm{~N}$ data is $O\left(e P^{7}\right)$. Therefore many predictions for NNN electromagnetic observables can now be made. The last two columns of Table 3 shows results for the magnetic moments of the triton and Helium-3. The final number is quite close to the experimental result, but the convergence is a little odd. In particular, the short-distance $\mathrm{NN}$ operators at $O\left(e P^{4}\right)$ play a key role in shifting the predicted three-nucleon magnetic moments towards the experimental values. These operators are not present in the "standard nuclear-physics" calculations of, e.g. Ref. [26]. But they are clearly of considerable phenomenological importance: indeed the naive-dimensional analysis used here to 
power count $\mathbf{J}$ appears to under-state their contribution.

Ref. [27] used the same expansion to compute the threshold nd $\rightarrow \mathrm{t} \gamma$ cross section, and the photon polarization observable $R_{c}$. A wave function derived from the INOY potential gives:

$$
\begin{gathered}
\sigma_{n d}=0.279+0.044(25)+0.175(3) \mathrm{mb}=0.498(3) \\
\mathrm{eP} \quad \mathrm{eP}^{2} \quad \mathrm{eP}^{4}
\end{gathered}
$$

The importance of the short-distance NN M1 operators is again clear, although the convergence of this observable is peculiar partly because nd $\rightarrow \mathrm{t} \gamma$ is a "suppressed process". The result is in reasonable agreement with the experimental number of $0.508(15) \mathrm{mb}$, given the expected size of $O\left(e P^{5}\right)$ contributions.

Ref. [27] also showed that both $\sigma_{n d}$ and $R_{c}$ are quite sensitive to the doublet nd scattering length. This may explain why it is difficult to reproduce $\sigma_{n d}$ with the AV18 + UIX two-nucleon and three-nucleon forces, but a pionless EFT calculation [41] does an excellent job with both.

An important test of these current operators will be their ability to describe the $|\mathbf{q}|^{2}$ dependence of observables. Therefore future computations of deuteron and tri-nucleon electro-disintegration will test the accuracy of the current operators derived in $\chi \mathrm{ET}$ - and of the $\chi \mathrm{ET}$ wave functions.

\section{On to the four-body system....}

Lazauskas et al. extended their computation of threshold M1 capture to the $n^{3} \mathrm{He}$ case in Ref. [28]. The results with different $4 \mathrm{~N}$ wave functions bracket the experimental number. It will be interesting to see what a study of this process with consistent $4 \mathrm{~N} \chi \mathrm{ET}$ wave functions, computed up to $O\left(P^{4}\right)$, reveals. In particular, the short-distance current operators at $O\left(e P^{4}\right)$ produce a large fraction of the total threshold cross section (see also Ref. [42]). The final results of Refs. [28, 42] for ${ }^{3} \mathrm{He}(\mathrm{n}, \gamma)^{4} \mathrm{He}$ at this order then exhibit pronounced $\Lambda$-sensitivity, suggesting that they may be missing some short-distance dynamics.

At higher energies the photon response of the Helium- 4 nucleus is dominated by the E1 transition. This makes the computation of Helium-4 photodisintegration relatively straightforward, as Siegert's theorem can be used to incorporate the exchange currents needed to maintain current conservation. While a systematic study would use the full NN current operator, this Siegert-theorem approach already indicates that the $\chi$ ET wave functions hold promise for describing Helium-4 photodisintegration data [29].

\section{A word on weak processes}

Some of the most dramatic progress in few-nucleon-system calcuations using $\chi$ ET has come in the arena of weak processes. Ab initio calculations of neutrino scattering up to several orders in the expansion for systems as large as $A=4$ have been carried out $[43,44]$. The $\chi$ PT expansion yields such a precise result for the weak current that the extent of the agreement between the very accurate experimental result for the rate of muon capture on Helium-3, $\Gamma=1496(4) \mathrm{Hz}$, and the theory prediction, $\Gamma=1499$ (16) Hz, constrains the conserved-vector-current hypothesis [45]. 
Meanwhile, two novel results for two-body weak currents have recently been obtained in $\chi \mathrm{ET}$. First, it was shown that a short-range axial current which makes a small, but significant, contribution to the tritium beta-decay rate is connected to a piece of the chiral three-nucleon force [46]. This relationship follows from the two-body analog of the Goldberger-Trieman relation, and provides the best constraint obtained thus far on the LEC $c_{D}$ that appears in the three-nucleon force [47].

Second, the relative importance of this shorter-distance beta-decay two-body current and the more-standard pion-range two-body current varies in different nuclei. There is a recent claim that this interplay helps explain discrepancies between previous computations of the Helium- 6 betadecay rate and experiment [48]. It is thus possible that the shifting balance between different aspects of the $\chi \mathrm{ET}$ axial current operator is ultimately responsible for the "quenching of $g_{A}$ " that is observed when single-particle models are used to describe beta-decay rates in larger nuclei.

If this explanation proves correct, it will be a huge success for the $\chi \mathrm{ET}$ approach. As in the case of the tri-nucleon magnetic moments, $\chi \mathrm{ET}$ is able to describe these weak decays in fewnucleon systems because it includes all mechanisms that can contribute to the current operator at a given order in the expansion, and so correctly accounts for one-body mechanisms, pion-range two-body currents, and shorter-distance two-body currents.

\section{Acknowledgments}

This research was supported by the U. S. Department of Energy, Office of Nuclear Physics, under contract No. DE-FG02-93ER40756 with Ohio University, and through the Mercator Programme of the Deutsche Forschungsgemeinschaft. I thank the organizers for a stimulating workshop, and my interlocutors and collaborators for many interesting discussions on these issues.

\section{References}

[1] S. Weinberg, Phys. Lett. B 251, 288 (1990); Nucl. Phys. B 363, 3 (1991).

[2] C. Ordonez, L. Ray and U. van Kolck, Phys. Rev. C 53, 2086 (1996).

[3] E. Epelbaum, W. Glöckle, and U.-G. Meißner, Nucl. Phys. A 671, 295 (2000).

[4] D. R. Entem and R. Machleidt Phys. Lett. B 524, 932002.

[5] D. R. Entem and R. Machleidt, Phys. Rev. C 68, 041001(R) (2003).

[6] E. Epelbaum, W. Glöckle and U-G. Meißner, Nucl. Phys. A 747, 362 (2005).

[7] S. R. Beane, P. F. Bedaque, M. J. Savage, and U. van Kolck, Nucl. Phys. A 700, 377 (2002).

[8] D. Eiras and J. Soto, Eur. Phys. J. A 17, 89 (2003).

[9] A. Nogga, R.G.E. Timmermans and U. van Kolck, Phys. Rev. C 72, 054006 (2005).

[10] M. C. Birse, Phys. Rev. C 74, 014003 (2006).

[11] M. Pavon Valderrama and E. Ruiz Arriola, Phys. Rev. C 74, 064004 (2006) [Erratum-ibid. C 75, 059905 (2007)].

[12] C.-J. Yang, Ch. Elster and D. R. Phillips Phys. Rev. C 77, 014002 (2008).

[13] E. Epelbaum, PoS (CD09), 077. 
[14] M. C. Birse, PoS (CD09), 078.

[15] C.-J. Yang, Ch. Elster, and D. R. Phillips, PoS (CD09), 064.

[16] G. P. Lepage, arXiv:nucl-th/9706029.

[17] E. Epelbaum and Ulf-G. Meißner, arXiv:nucl-th/0609037.

[18] U. van Kolck, Phys. Rev. C 49, 2932 (1994).

[19] E. Epelbaum et al., Phys. Rev. C 66, 064001 (2002).

[20] E. Epelbaum, arXiv:nucl-th/0511025; D. Rozpedzik et al., Acta Phys. Polon. B 37, 2889 (2006).

[21] M. Walzl and U.-G. Meißner, Phys. Lett. B 513, 37 (2001).

[22] D. R. Phillips, Phys. Lett. B 567, 12 (2003).

[23] D. R. Phillips, J. Phys. G 34, 365 (2007).

[24] M. Pavon Valderrama, A. Nogga, E. Ruiz Arriola and D. R. Phillips, Eur. Phys. J. A 36, 315 (2008).

[25] Y. H. Song, R. Lazauskas, T. S. Park and D. P. Min, Phys. Lett. B 656, 174 (2007).

[26] L. E. Marcucci, M. Pervin, S. C. Pieper, R. Schiavilla and R. B. Wiringa, arXiv:0810.0547 [nucl-th].

[27] Y. H. Song, R. Lazauskas and T. S. Park, Phys. Rev. C 79, 064002 (2009).

[28] R. Lazauskas, Y. H. Song and T. S. Park, arXiv:0905.3119 [nucl-th].

[29] S. Quaglioni and P. Navratil, Phys. Lett. B652, 370 (2007).

[30] J. A. McGovern, H. W. Grießhammer, D. R. Phillips, and D. Shukla, PoS (CD09), 059.

[31] H. W. Grießhammer and D. Shukla, PoS (CD09), 060.

[32] D. Choudhury, A. Nogga and D. R. Phillips, Phys. Rev. Lett. 98, 232303 (2007).

[33] D. Shukla, A. Nogga and D. R. Phillips, Nucl. Phys. A 819, 98 (2009).

[34] M. Pavon Valderrama and E. Ruiz Arriola, Phys. Rev. C 72, 054002 (2005).

[35] D. Abbott et al. [JLAB t20 Collaboration], Eur. Phys. J. A 7, 421 (2000).

[36] D. M. Nikolenko et al., Phys. Rev. Lett. 90, 072501 (2003).

[37] S. Kölling, E. Epelbaum, H. Krebs and U.-G. Meißner, arXiv:0907.3437, Phys. Rev. C (in press).

[38] T.-S. Park, N. Kaiser, D. R. Phillips, in preparation.

[39] T. S. Park, K. Kubodera, D. P. Min and M. Rho, Phys. Lett. B 472, 232 (2000).

[40] S. Pastore, L. Girlanda, R. Schiavilla, M. Viviani and R. B. Wiringa, Phys. Rev. C 80, 034004 (2009);

S. Pastore, R. Schiavilla and J. L. Goity, Phys. Rev. C 78, 064002 (2008).

[41] H. Sadeghi, S. Bayegan, H. W. Grießahmmer, Phys. Lett. B 643, 263 (2006).

[42] R. Schiavilla, talk at 19th International Conference on Few-body Problems in Physics, Bonn, Germany, August 2009. To appear in the proceedings.

[43] T. S. Park et al., Phys. Rev. C 67, 055206 (2003).

[44] D. Gazit and N. Barnea, Phys. Rev. Lett. 98, 192501 (2007); Nucl. Phys. A 790, 356 (2007).

[45] D. Gazit, Phys. Lett. B 666, 472 (2008).

[46] A. Gårdestig and D. R. Phillips, Phys. Rev. Lett. 96, 232301 (2006).

[47] D. Gazit, S. Quaglioni and P. Navratil, Phys. Rev. Lett. 103, 102502 (2009).

[48] S. Vaintraub, N. Barnea and D. Gazit, Phys. Rev. C 79, 065501 (2009). 